\title{
Bilingualism and the Emotional Intensity of Advertising Language
}

\author{
STEFANO PUNTONI \\ BART DE LANGHE \\ STIJN M. J. VAN OSSELAER*
}

\begin{abstract}
This research contributes to the current understanding of language effects in advertising by uncovering a previously ignored mechanism shaping consumer response to an increasingly globalized marketplace. We propose a language-specific episodic trace theory of language emotionality to explain how language influences the perceived emotionality of marketing communications. Five experiments with bilingual consumers show (1) that textual information (e.g., marketing slogans) expressed in consumers' native language tends to be perceived as more emotional than messages expressed in their second language, (2) that this effect is not uniquely due to the activation of stereotypes associated to specific languages or to a lack of comprehension, and (3) that the effect depends on the frequency with which words have been experienced in native- versus second-language contexts.
\end{abstract}

$\mathrm{E}$ nglish is the new lingua franca. From international business to the Internet and from science to music, English is the language of important aspects of the social life of consumers around the world (Cristal 1997). Indeed, it has become commonplace for commentators to identify the rise of English as a world language as one of the most visible aspects of the process of globalization. No area of media production exemplifies the growing importance of the English language better than advertising. Regardless of their cultural heritage and native language, consumers are routinely addressed by large numbers of marketing messages in English. For example, in the Netherlands over $40 \%$ of TV ads contain words in English (Gerritsen et al. 2000), and this phenomenon is by no means limited to Western cultures (e.g., Lee 2006).

Calls for an increased focus on the consequences of globalization for consumers (e.g., Johar, Maheswaran, and Peracchio 2006) emphasize the need to improve the current

*Stefano Puntoni (spuntoni@rsm.nl) is an assistant professor of marketing, Bart de Langhe (blanghe@rsm.nl) a PhD student, and Stijn M. J. van Osselaer (sosselaer@ rsm.nl) a professor of marketing at the Rotterdam School of Management, Erasmus University, PO Box 1738, 3000 DR Rotterdam, the Netherlands. The authors thank Patricia Heijndijk, Malini Sietaram, Steven Sweldens, Mirjam Tuk, Ralf van der Lans, Joëlle Vanhamme, and Peeter Verlegh for their help with stimulus development and data collection and Maria Galli, Martijn de Jong, and Nader Tavassoli for their useful comments. The financial support of the Erasmus Research Institute of Management is gratefully acknowledged.

John Deighton served as editor and Laura Peracchio served as associate editor for this article.

Electronically published October 27, 2008 understanding of how the globalization of advertising language influences consumer response to advertising messages. In particular, no previous research has examined the emotional consequences of the use of a foreign language in marketing messages. Generating emotional experiences around a brand is an important goal of brand communication. For instance, French Connection, a British fashion company, adopted the acronym FCUK in all its advertising, presumably in the belief that provocative messages such as "FCUK you!" are beneficial to the brand. The globalization of advertising implies that marketing messages are increasingly delivered in a language that is different from consumers' native tongue. For example, the acronym FCUK has been advertised to consumers in over 20 countries where English is not an official language. This raises the question of whether and how language might affect consumer perceptions of ad campaigns aimed at triggering emotional reactions.

The field of linguistics is displaying a growing awareness of the role of emotional processes in bilingualism (Pavlenko 2005, 2006). Extending recent literature on the emotions of bilinguals, this article investigates the perceived emotionality of marketing messages in consumers' native language (L1) versus second language (L2). We present five experiments showing (1) that messages expressed in L1 tend to be perceived as more emotional than messages expressed in L2, (2) that this effect is not uniquely due to the activation of stereotypes associated to specific languages or to a lack of comprehension, and (3) that the effect depends on the frequency with which words have been experienced in L1 versus L 2 contexts. Together, these experiments provide sup- 
port for a language-specific episodic trace theory of language emotionality.

\section{THEORY}

Consumer research on bilingualism can be broadly categorized in two areas. The first adopts a sociolinguistic approach to examine the signaling functions of language in the context of ethnic minority targeting (e.g., Koslow, Shamdasani, and Touchstone 1994; Luna and Peracchio 2005). The second adopts a psycholinguistic approach to explore the information-processing consequences of language (e.g., Luna and Peracchio 2001; Tavassoli and Lee 2003). The present investigation shares with this second stream of research the stress on psycholinguistic processes but differs from existing consumer research in its attention to emotional processes.

\section{Existing Research on the Emotions of Bilinguals}

The prediction that one's native language possesses special emotional qualities is probably not surprising to any introspective bilingual. Despite the intuitive appeal of this prediction, the generality of the effect, and the obvious substantive implications, to the best of our knowledge no contribution in marketing has explored the possibility of systematic differences in the emotional intensity of marketing communication as a function of language. In other fields of inquiry, however, it is possible to find some evidence consistent with this intuition.

Linguistic research using introspection, interviews, or literary analysis has on a number of occasions mentioned the special emotionality of one's native language (Pavlenko 2005). Moreover, using both general self-reports (Dewaele 2004) and physiological measures (Harris, Aycicegi, and Gleason 2003), psycholinguistic research on taboo words and swearwords has shown that the perceived emotional intensity of these highly emotional words is greatest in one's native language. Research on code switching is also relevant to this discussion. In the context of social interaction, Bond and Lai's (1986) participants found it easier to discuss embarrassing topics in L2 than in L1. Bond and Lai argue that in embarrassing situations switching to a second language serves a distancing function. Based on clinical case studies, Javier (1989) similarly concluded that during therapy sessions switching language is a coping mechanism for the patient.

In these studies L1 yielded stronger emotional experiences than L2. These settings, however, are all characterized by a combination of extreme emotionality and self-relevance that makes the extrapolation to the processing of external information of mild emotionality questionable. In addition to the issue of the applicability of results on taboo words and bilingual counseling to a marketing setting, another important open issue is the mechanism responsible for systematic differences in language emotionality.

First, as most research in the area has focused on a specific language comparison (often featuring the language of a "warm" culture such as Spanish or Turkish as L1 and English as L2), it could be argued that most of the work on which evidence for the emotional advantage of L1 rests is open to a possible alternative explanation based on the (perceived) emotionality of specific languages. If country stereotypes influence language emotionality (Leclerc, Schmitt, and Dubé 1994), evidence for the emotional advantage of L1 must be produced in a context that controls for this influence, for example, by varying across respondents which of two languages is L1 versus L2 or by demonstrating moderating effects drawn from a theory of language emotionality.

Moreover, it is important to demonstrate that language effects on emotionality cannot only be explained by differences in comprehension between L1 and L2. Linguistic experience is an important determinant of a person's ability to interpret and appraise emotional expression (Harris 2000). For example, native speakers perform better than nonnative speakers when asked to identify verbal emotional expression (Pavlenko 2005). This is an especially important issue when shifting attention from single words and isolated utterances to more complex textual information such as advertising slogans or product descriptions.

Recently, a number of authors have argued that differences in the relative emotionality of L1 and L2 must stem from differences in the context of language learning and use (Altarriba 2003; Harris, Gleason, and Aycicegi 2006; Pavlenko 2005). For example, Dewaele (2004) showed that languages learned in an instructed context are associated with less intense emotional reactions than languages learned in a naturalistic context. Below, we propose a cognitive model of bilingual memory that builds on this literature and on episodic trace theories of memory.

\section{A Language-Specific Episodic Trace Theory of Language Emotionality}

Episodic trace models of memory represent one of the most influential theoretical traditions within cognitive psychology (Raaijmakers and Shiffrin 1992). They are founded upon the assumption that every experience leaves a separate episodic trace in memory. Consistent with nonanalytic views of cognition (Jacoby and Brooks 1984), these models posit that perceptual and contextual details of experiences are stored in memory and are integral to later perception. For example, Hintzman's Minerva 2 model (Hintzman 1986, 1988) suggests that each experience is stored as an array of elements. When a stimulus is encountered, all memory traces are activated in proportion to their similarity to the probing stimulus. An aggregate of all activated traces (i.e., an echo) is sent to working memory from long-term memory. The echo may contain information that is not present in the stimulus, such as previously experienced emotions, thus associating the stimulus to past emotional experiences. For episodic memories to influence emotional reactions, the actual conscious retrieval of such episodes is not necessary. The emotional intensity resulting from the echo content ac- 
tivated by the probing stimulus is experienced as an intuitive impression or gut feeling (Schimmack and Reisenzein 1997; Siemer and Reisenzein 2007).

Similarity to Episodic Traces. Episodic trace theories propose that "surface" details of experiences are stored in memory as elements of the episodic trace. Consistent with this reasoning, it has been found that auditory details, like intonation contour and vocal pitch (Palmeri, Goldinger, and Pisoni 1993; Schacter and Church 1992), and irrelevant visual information, like typeface (Jacoby and Hayman 1987), are implicitly stored in long-term memory. Hence, it seems plausible that these episodic traces also contain L1 or L2 lexical representations depending on the language in which the event was originally experienced. This property of episodic memory can explain language-dependent recall, the finding that memories originally experienced in L1 (L2) are more accessible when triggered by L1 (L2) words (Marian and Kaushanskaya 2004; Marian and Neisser 2000). This research stream builds on the encoding specificity principle (Tulving and Thomson 1973) and argues that experiences are stored in memory together with their linguistic context. Activating the same linguistic representation that was used at the time of the experience serves as a retrieval cue for memories for that experience. Given the centrality of emotional information in autobiographical memories (Bower 1981), we draw on the idea of language-dependent storage of autobiographical memories. For example, research has shown that individuals tend to express more emotional details and more intense affect when the language of the retrieval cue is consistent with the language at encoding (Javier, Barroso, and Munoz 1993; Marian and Kaushanskaya 2004). Our theory embeds the principle of language-dependent recall within a cognitive model and extends this research from the conscious recollection of autobiographical memories to the perception of stimuli in which emotionality is automatically attributed to textual information based on implicit language-specific activation of episodic traces stored in memory. In sum, we propose that textual information such as marketing slogans may function as memory probes that lead to the activation, and feeling, of emotions experienced before in same-language contexts.

Number of Episodic Traces. Episodic trace theories suggest that, in general, the activation intensity of an echo is a positive function of the number of relevant episodic traces stored in memory (Hintzman 1986). Words that are encountered more often should be part of a larger number of episodic traces, leading to a stronger echo of emotions that have been experienced during these episodes. As a result, there should be a positive correlation between how often a word is encountered in a particular language and emotionality. In the context of the globalization of advertising language, the number of events experienced by consumers in concomitance with an L1 language context generally outnumbers that of events experienced in an L2 language context. Together with the previous property of episodic traces, this discussion therefore leads to the pre- diction that marketing communication should in general trigger stronger emotional responses in L1 than in L2. Moreover, our theory predicts an emotional advantage of L2 in the (rare) case of words that are experienced more often in L2 language contexts.

Lexical Representations. In addition to separate storage of individual experiences (Hintzman 1986), we adopt the assumption of most psycholinguistic models of bilingual memory, such as the Revised Hierarchical Model (Kroll and Stewart 1994), of the existence of independent lexical stores for L1 and L2. Lexical stores encode the lexical representation of words (i.e., how they are written and how they sound). Moreover, we adopt the contention of prior psycholinguistic literature (e.g., Kroll and Stewart 1994) that the link between the lexical representation of L1 and L2 words is asymmetric. Specifically, the link from L2 words to their L1 translation is stronger than the link in the opposite direction. In other words, the activation of an L1 word as a consequence of exposure to its L2 translation is greater than that of an L2 word as a consequence of exposure to its L1 translation. Thus, consumers may unconsciously translate L2 words into L1 words but will rarely do the opposite.

Our model incorporates the interaction between episodic memory traces and lexical representations. A prediction of the theory is that the emotional intensity generated by L2 probes benefits to some extent from the association between the L2 probe and its L1 translation. In other words, L2 probes activate emotional echo content from L2 experiences as well as emotional echo content from L1 experiences, proportionally to the level of activation of L1 lexical representations. As corollaries of the theory, this discussion implies that (1) when the accessibility of the L1 translation is increased, L2 words should be perceived as more emotional and, conversely, that (2) when this indirect activation of L1 emotional echo content upon presentation of L2 words is impeded, the emotional advantage of L1 should become larger.

In sum, the language-specific episodic trace theory assumes that there are two routes to perceived emotionality for marketing messages. In the direct route, L1 (L2) words trigger episodic memory traces experienced in an L1 (L2) context. Because there are usually more L1 than L2 traces, marketing messages in L1 tend to be perceived as more emotional than those in L2. In the indirect route, words presented in L2 partially activate the corresponding words in the L1 lexical store. Because these L1 words, in turn, function as probes for L1 episodic traces, L2 words can benefit to some extent from the emotionality of the experiences triggered by L1 words. This reduces the difference in the perceived emotionality of L1 and L2 words. Figure 1 presents a summary of the theory.

\section{STUDY 1}

Study 1 was designed to test the prediction of the theory that advertising information in L1 tends to be experienced 
FIGURE 1

LANGUAGE-SPECIFIC EPISODIC TRACE THEORY OF LANGUAGE EMOTIONALITY

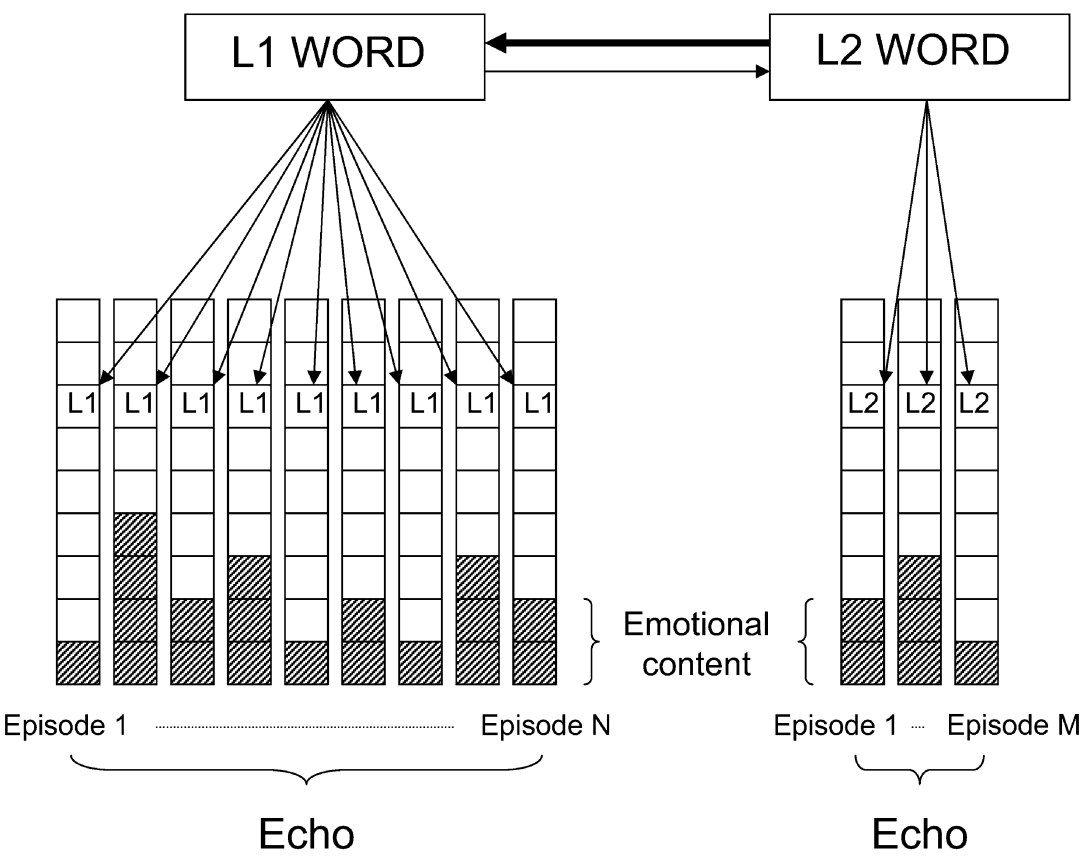

as more emotional than the same information in L2. As target textual information, we used a series of advertising slogans. To establish the emotional advantage of L1 in a context that ensured its validity, we tested the effect of language on perceived emotionality (1) in conjunction with an assessment of an alternative complex appraisal (perceived originality) and (2) in a balanced bilingual design that varied across participants which of two target languages was L1 versus L2.

The first aspect above was included to show that the advantage of L1 is not general to all complex appraisals. We chose perceived originality because it is not likely to be a natural part of most consumer experiences. Thus, whereas emotional content is central in episodic memory, most memory traces of previous experiences are unlikely to contain originality judgments. In other words, we predict a two-way interaction between language and appraisal such that L1 stimuli will be rated as more emotional than L2 stimuli in the absence of a similar effect of language on originality.

The second aspect above, varying which of two languages was L1 versus L2, was necessary to rule out a languagespecificity explanation that haunts many studies on bilinguals, in which the language used as L1 is different across all respondents from the language used as L2. We address this problem by using the same two languages and manipulating the role they played (L1 or L2). Specifically, we used Dutch-French bilinguals, exactly half of whom were French native speakers (L1) who had learned to speak Dutch (L2). The other half were Dutch native speakers (L1) who had learned to speak French (L2). Thus, any difference be- tween the emotionality of messages in L1 and the emotionality of messages in L2 could not be explained by, for example, the greater emotionality of Romance languages such as French over Germanic languages such as Dutch. An additional aspect of the design deserves attention. To obtain the cleanest test of the effect of language on emotionality, the study used trilingual participants. This allowed instructions and experimenter-participant interactions to occur in a language (English) that was different from the target languages used for the stimuli, hence avoiding potential asymmetric effects of these interactions across people with different L1s.

\section{Method}

Design and Participants. The study used a 2 (language of slogans: L1 vs. L2) $\times 2$ (type of appraisal: emotional intensity vs. originality) $\times 2$ (L1: native French speakers vs. native Dutch speakers) mixed design, in which language of slogans and type of appraisal were within-subjects factors, and respondents' L1 was a between-subjects factor. In addition, the order of the slogans and the language sequence were counterbalanced between subjects. The experiment was conducted in Brussels, the bilingual FrenchDutch capital city of Belgium. Respondents were 64 DutchEnglish-French trilinguals who participated in return for a small reward (for age, $M=25.48 ; \mathrm{SD}=7.06 ; 25$ females).

Stimuli. The slogans had been created using as a starting point American slogans unknown in Europe. The slogans 
TABLE 1

CELL MEANS (AND STANDARD DEVIATIONS) IN STUDY 1

\begin{tabular}{|c|c|c|c|c|c|c|c|c|}
\hline & \multicolumn{4}{|c|}{ L1 } & \multicolumn{4}{|c|}{ L2 } \\
\hline & \multicolumn{2}{|c|}{ French native speakers } & \multicolumn{2}{|c|}{ Dutch native speakers } & \multicolumn{2}{|c|}{ French native speakers } & \multicolumn{2}{|c|}{ Dutch native speakers } \\
\hline & Females & Males & Females & Males & Females & Males & Females & Males \\
\hline Emotional intensity & $\begin{array}{c}4.58 \\
(.87)\end{array}$ & $\begin{array}{c}4.48 \\
(.98)\end{array}$ & $\begin{array}{c}4.74 \\
(1.24)\end{array}$ & $\begin{array}{c}4.59 \\
(.90)\end{array}$ & $\begin{array}{c}4.14 \\
(1.14)\end{array}$ & $\begin{array}{c}4.28 \\
(1.08)\end{array}$ & $\begin{array}{l}4.21 \\
(.96)\end{array}$ & $\begin{array}{c}4.48 \\
(.89)\end{array}$ \\
\hline Originality & $\begin{array}{c}3.55 \\
(1.25)\end{array}$ & $\begin{array}{c}4.22 \\
(1.09)\end{array}$ & $\begin{array}{c}3.69 \\
(1.26)\end{array}$ & $\begin{array}{c}3.30 \\
(1.11)\end{array}$ & $\begin{array}{c}3.61 \\
(1.40)\end{array}$ & $\begin{array}{c}3.80 \\
(1.16)\end{array}$ & $\begin{array}{c}3.62 \\
(.99)\end{array}$ & $\begin{array}{c}3.72 \\
(1.20)\end{array}$ \\
\hline
\end{tabular}

NOTE. $-\mathrm{L} 1$ = native language; $\mathrm{L} 2$ = second language.

were therefore created in English and later translated to Dutch and French by native Dutch and French speakers. The translated slogans were then checked for consistency with the English version by two independent judges. Each slogan was presented together with an indication of the product or service advertised (in English for all respondents). The slogans spanned a variety of product categories: flowers ("See the face you love light up in a brilliant smile"), hotel ("When you are here you are family. We will leave the light on for you"), amusement park ("Where a kid can be a kid and the magic never ends. The happiest place on Earth"), frozen food ("Nothing comes closer to home. Be happy, be healthy!"), antidrug campaign ("Parents who use drugs have kids who use drugs"), and construction toys ("Build something together with your child. You will never outgrow our toys"). The six slogans, together with their product category, were presented on one page.

Two slogan orders were randomly created, and two additional orders were generated by inverting the first two, resulting in four different slogan orders. For the language sequences, the same procedure was applied. The four slogan orders were then crossed with the four language sequences, resulting in 16 different counterbalancing conditions.

Procedure. The city of Brussels was selected as the setting for the study because of its special linguistic characteristics. Belgium includes four language regions: Dutchspeaking, French-speaking, German-speaking, and the bilingual region of Brussels. Many people living and working in Brussels are fluent in both French and Dutch. Participants were recruited in the three main subway and train stations in Brussels. Train travelers were addressed in English, asked what their native language was (Dutch or French), and asked whether they were fluently trilingual (English, Dutch, and French). If they indicated they were fluently trilingual, they were invited to participate in an international study on advertising slogans.

Participants were asked to complete a booklet with instructions and questions in English. The second page presented six slogans, three in French and three in Dutch. Participants were asked to rate the emotional intensity of the slogans, using a 7-point unipolar scale (anchoring points were "unemotional" and "emotional"). The following page featured again the same slogans and asked participants to rate their perceived originality (anchoring points were "unoriginal" and "original"). We chose single-item measures because they allowed us to include multiple slogans and both emotionality and originality judgments without inducing fatigue or resistance to participate in a public transport setting.

\section{Results}

For all participants, the emotionality and originality scores of the slogans in each language were averaged to form four indexes. The data were subjected to a repeated-measures ANCOVA with language of the slogan (L1 vs. L2) and type of appraisal (emotionality vs. originality) as within-subjects factors and respondents' L1 as a between-subjects factor. Gender has been shown to affect sensitivity to emotional information (Bloise and Johnson 2007), and this variable was therefore added as an additional factor. Slogan order, language sequence, and their interaction were added as covariates. See table 1 for means and figure 2 for a representation of the language by appraisal interaction.

We observed no main effect of respondents' L1 $(p>.74)$, a marginally significant main effect of language $(F(1,45)=$ $3.05, p<.09$; ratings of L1 slogans were marginally higher than ratings of L2 slogans), and a significant main effect of type of appraisal $(F(1,45)=45.74, p<.0001$; emotionality ratings were generally higher than originality ratings). Crucially, the two-way interaction between language of slogans and type of appraisal was significant $(F(1,45)=4.15, p<$ .05). This interaction was in the expected direction. The L1 slogans $(M=4.59)$ were rated as more emotional than L2 slogans $(M=4.30 ; F(1,45)=6.86, p=.01)$. No effect of language was observed on ratings of originality $(p>.9$; for $\mathrm{L} 1, M=3.72$; for $\mathrm{L} 2, M=3.70$ ). This two-way interaction was not qualified by the three-way interaction between respondent's L1, language, and type of appraisal $(p>.36)$. In particular, the mean emotional advantage of L1 was 0.29 for native speakers of both French (for L1, $M=4.52$, and for $\mathrm{L} 2, M=4.23)$ and Dutch ( $M=4.65$ and 4.36, respectively). In other words, regardless of whether the native language of participants was French or Dutch, the emotional advantage of L1 was identical.

The three-way interaction between language of the slogan, appraisal, and gender was marginally significant $(F(1,45)=$ 
FIGURE 2

LANGUAGE BY APPRAISAL INTERACTION IN STUDY 1

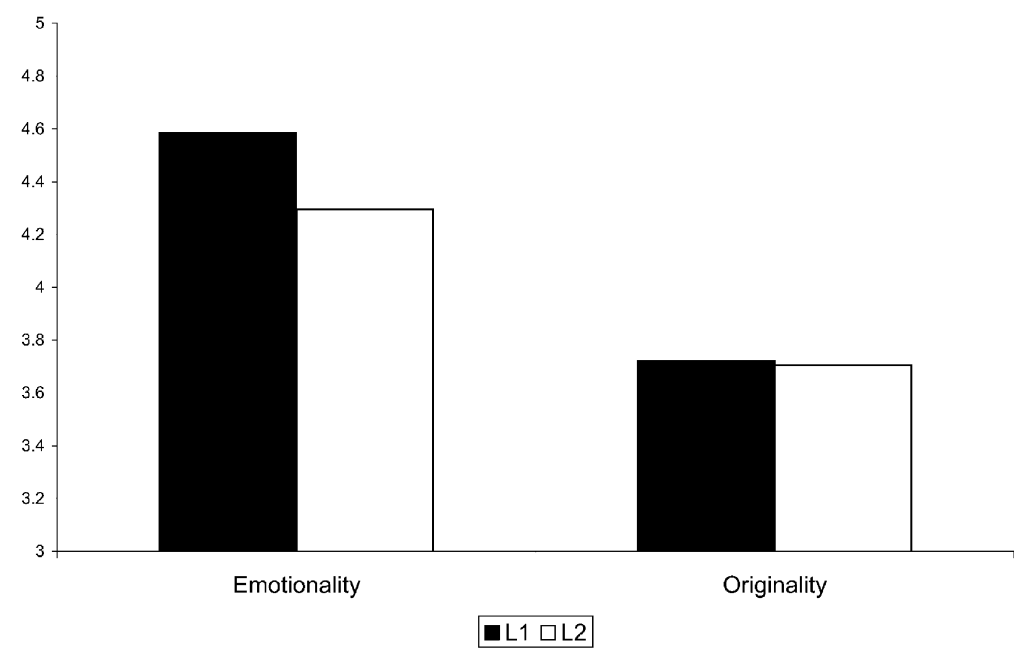

$2.99, p<.1)$. The emotional advantage of L1 was more pronounced for women $(M=0.49)$ than men $(M=0.16$; $F(1,45)=5.99, p=.02)$, with no language by gender effect on originality $(p>.78)$. Research on autobiographical memories suggests that females have stronger memory for emotional events than males (Davis 1999), and this difference might explain this marginally significant effect. A number of theoretically uninteresting effects related to the counterbalancing covariates (e.g., effects of slogan order) were also significant.

\section{Discussion}

In this study, Dutch-English-French trilinguals were addressed in English and rated a series of Dutch and French advertising slogans on emotionality and originality. Half of the participants were L1 speakers of French, and half were L1 speakers of Dutch. We observed a two-way interaction between language and type of appraisal such that L1 slogans were rated as more emotional than L2 slogans, with no difference between L1 and L2 slogans in perceived originality. The effect on emotionality confirms our hypothesis. The absence of an effect on originality suggests that the L1 advantage is not a universal characteristic of all complex appraisals but is due to the centrality of affect in episodic memory. The two-way interaction, moreover, was not qualified by a three-way interaction with respondents' L1: the magnitude of the emotional advantage of L1 was exactly the same for Dutch and French native speakers. Thus, the L1 advantage on emotionality could not be due to either of the languages being inherently more emotional.

\section{STUDY 2}

Study 1 provided a stringent test of the difference in the emotionality of L1 and L2. However, L1 and L2 differ in more respects than just the number of previous experiences in which words were paired with emotions. For example, it is possible that consumers often do not (completely) comprehend emotion-related words in L2. If words are not comprehended and do not strongly activate any specific meaning, it is likely that they do not generate high judgments of emotionality. If words are miscomprehended and activate incorrect concepts, it is likely that the activated concepts are not all as emotional as the ones that should have been activated, assuming that most slogans are chosen to be at least somewhat emotional. Thus, miscomprehension could lead to more moderate emotionality judgments in L2.

Study 2 was designed to address this concern. Participants rated the perceived emotionality of either L1 or L2 single words. The critical feature of the study is that all target words are perceptually similar across language conditions and share the same meaning-they are cognates. Cognates (e.g., the English "emotional" and Dutch "emotioneel") are easy to process and comprehend in L2, a phenomenon often referred to as the cognate facilitation effect (Costa, Caramazza, and Sebastian-Galles 2000). Thus, difficulty comprehending the target words is unlikely to explain differences in emotional intensity.

\section{Method}

In this study, we manipulated the language in which eight words were presented in a simple two-cell design. Participants were 79 undergraduate students enrolled in programs taught partially or completely in English at Erasmus University. They were all native Dutch speakers and participated in return for course credits (for age, $M=19.87$; $\mathrm{SD}=$ 1.94; 33 females). The eight words were selected based of the similarity between L1 and L2. In all cases, no more than two letters differed between the L1 and L2 words, and for all target words the L1 and L2 pronunciation matched 
closely. The L2 stimuli were "depression," "heroic," "house," "intimate," "mother," "poetic," "relation," and "sick." The L1 stimuli were "depressie," "heroisch," "huis," "intiem," "moeder," "poetisch," "relatie," and "ziek." Participants were randomly assigned to a condition and completed this study in individual cubicles as part of a sequence of unrelated experiments. The words were presented on one screen, and participants were asked to indicate to what extent each word had emotional connotations (on a 7-point scale, from "no emotional connotations" to "strong emotional connotations").

\section{Results and Discussion}

We found a significant main effect of language $(F(1,77)=$ 4.98, $p<.05)$. Despite the fact that the L1 and L2 words were virtually identical, participants who saw the words in L1 $(M=4.66)$ indicated that the words had stronger emotional connotations than participants who saw the words in L2 indicated $(M=4.33)$. Another model was estimated with gender as an additional factor. The main effect of language remained unchanged, and the main effect of gender was significant $(F(1,75)=4.46, p<.05$; females rated the words as more emotional than males did). The interaction between gender and language was, however, nonsignificant $(p>.56)$. Using a different experimental paradigm, study 2 corroborates the evidence in support of the emotional advantage of L1 and against an explanation purely in terms of inability to comprehend the stimuli in L2.

\section{STUDY 3}

Based on the indirect route to the emotionality of L2 words, we predicted that, when the L1 translation of an L2 probe is made more accessible, the emotional intensity of the L2 probe should benefit from the increased activation of emotional echo content from L1 experiences. Study 3 was designed to test the prediction that the emotionality of L2 words increases when the equivalent L1 word is made more accessible. In addition, study 3 adds to the evidence obtained in study 2 against an explanation of language effects on emotionality only based on comprehension differences.

In study 3 we developed a novel experimental paradigm, which allowed assessing the effect of the activation of L1 versus L2 words while holding constant across conditions the language of the stimuli (in this study, single words presented in English). Instead of varying the language of presentation of target words, we exposed all participants to only their L2 version and manipulated the accessibility of the L1 representation of those L2 words. The experiment was presented as a study on the effect of handwriting on the evaluation of words used in advertising. Participants wrote down on a piece of paper a series of target English words and associations with those words using either their native language or English.

In contrast to an explanation based on ability to comprehend, a multiple-trace episodic memory view does predict an L1 advantage in this study. When a word is presented and reproduced in L2, consumers are likely to follow the direct route and use the L2 word as an episodic memory probe. Because consumers have few emotional experiences stored with the L2 word in episodic memory, the emotional echo content will be relatively weak, leading to a moderate judgment of the word's emotionality. However, if consumers are asked to translate the L2 word to L1 and to generate associations in L1, they are likely to use the L1 word to probe their episodic memory, yielding a stronger emotional echo content because more experiences were stored with L1 words than with L2 words. Relative to a control condition, increasing the accessibility of their L1 translation should therefore increase the perceived emotionality of L2 words. This design has the additional advantage of enabling the inclusion of participants with a variety of native languages, hence, allowing a strong test of external validity.

\section{Method and Results}

Accessibility of native language was manipulated between subjects using two levels. Participants were 60 foreign undergraduate students (30 females) at Erasmus University who were all nonnative English speakers. In total, 20 different native languages were represented in the sample (e.g., Bulgarian, Mandarin, Croatian, German, Indonesian, Russian, and Spanish).

The target words were 12 English words ("birthday," "children," "family," "garden," "loneliness," "pain," "party," "play," "relation," "school," "sick," and "toothbrush"). They had been selected to represent a broad set of notions relating to everyday life (e.g., "toothbrush") and ranging on a number of dimensions, for example, from abstract (e.g., "loneliness") to concrete (e.g., "garden") and from positive (e.g., "play") to negative (e.g., "pain"). For each word, participants in the high native-language accessibility condition were asked to translate the word into their native language and to write down on a sheet of paper in front of them the translation together with three associations that the word brought to mind, also in their native language. Participants in the control condition went through the same procedure, but instead of translating the English word to their native language, they were instructed to copy the English word and to write down three associations to the word in English. After writing down each word, the English word was presented again on the computer screen. Participants then rated the extent to which this L2 word had emotional connotations for them. Participants indicated their opinion on a scale from 1 to 7 , with 1 meaning "no emotional connotations" and 7 meaning "very strong emotional connotations."

The emotionality scores for the 12 words were averaged for each participant to create an emotionality index. The main effect of accessibility of native language was significant and in the predicted direction $(F(1,58)=4.92, p<$ .05). Participants who wrote down the target words in their native language $(M=5.08)$ reported a stronger emotional response than participants who wrote down the words in English $(M=4.66)$. The analyses were repeated adding 
gender as an additional factor. No coefficient involving gender was significant in this model ( $p$ 's $>.24$ ), and the main effect of accessibility of native language was left unchanged.

\section{Discussion}

In study 3, all participants rated the emotionality of a series of English words. Before rating its emotionality, half were asked to write down the word in their native language and half in English. Consistent with a multiple-trace memory view of language and emotionality, we observed a significant main effect of this manipulation of L1 accessibility on emotionality ratings of L 2 words. Because the target stimuli were always presented in L2, the effect in this experimental paradigm cannot be explained by the inability to comprehend words in L2. In addition, participants in study 3 were citizens of a wide range of countries. This feature of the study is important because it allowed the inclusion of a large number of native languages, hence, adding to the external validity of the experiment.

\section{STUDY 4}

Studies 1-3 established the emotional advantage of L1 and together demonstrated that an account based on comprehension or language stereotypes cannot be solely responsible for the emotional advantage of L1. The data are consistent with the idea that words in a language are stored together with emotional content in episodic memory traces such that perceiving words in a language activates records of emotional experiences featuring those words in that language.

If this theory is correct, the language context in which emotions tend to be experienced should predict the relative emotionality of L1 versus L2. If the language context is a key determinant of emotionality, it should be possible to reverse the effect of language observed in previous studies for words that are predominantly experienced in an L2 context. In other words, the theory predicts that, for words that have been encountered mostly in an L2 language context, the effect of language should reverse, and L2 words should be perceived as more emotional than L1 words. Study 4 was designed to test this contention. As stimuli, we used Dutch (L1) and English (L2) word pairs selected through a pretest. In this experiment we predict no main effect of language but a crossover interaction such that, for words experienced predominantly in L1 language contexts, the L1 words will be perceived as more emotional than their L2 equivalent, with the opposite holding for words experienced predominantly in L2 language contexts.

\section{Method}

Design and Participants. The design of this study was a 2 (language: L1 vs. L2) $\times 2$ (language context: L1 vs. L2) fully within-subjects design. In addition, the order of language presentation was manipulated between subjects. Four counterbalancing conditions were also added to vary between subjects the order in which the words were presented, leading to eight different versions of the booklet. Participants were 94 students at Erasmus University (for age, $M=23.28$; $\mathrm{SD}=1.85 ; 41$ females) who participated in return for a chocolate bar.

Procedure. At the end of a lecture, students were asked to fill out a booklet in which eight concepts were presented sequentially. For each concept, participants rated the emotionality of the corresponding words in L1 and in L2. For example, for the concept "funeral/begrafenis," they rated the emotionality of both "funeral" and "begrafenis" (on a 9-point scale ranging from "not emotional at all" to "very emotional"). For half of the participants, the L1 word preceded the L2 word, and for half it followed the L2 word. After rating all words, a final page asked participants to report whether any of the L2 words were unfamiliar (one participant reported low familiarity on at least one word, but excluding data from this participant left the results unchanged) and some basic demographic questions and to write a short essay to guess the purpose of the study (no participant raised a suspicion about the purpose of the study, and, in particular, none noticed the presence of two sets of concepts).

Stimuli. To select the stimuli we conducted a pretest with 50 students (for age, $M=21.38$; $\mathrm{SD}=1.94$ ). The pretest used a format similar to that of the main study, but it asked participants to rate the extent to which, for a given L1/L2 word pair, the word had been experienced more often in an L1 or an L2 language context. Twenty-four L1/L2 word pairs were presented sequentially (the order of the words was varied between subjects using four different versions of the booklet), and, for each pair, participants answered the following item: "How often have you encountered this concept in a Dutch-language context or an English-language context?" (9-point scale, from "much more often in Dutch-language contexts" to "much more often in English-language contexts"). The pretested concepts had been selected with the goal of ensuring variance in the context of language use. Although the average across all concepts was significantly smaller than the midpoint of the scale (hence, indicating on average a predominantly Dutchlanguage context, $p<.0001$ ), we observed relatively large variance in language context mean ratings across the 24 concepts $(2.62<M<6.36 ; \mathrm{SD}=1.03)$. The scores were ranked, and the four word pairs that scored lowest $(M=$ $3.10)$ and the four that scored highest $(M=6.00, t(49)=$ $-12.97, p<.0001)$ were selected for the main study as, respectively, L1 and L2 language context word pairs. The L1 language context words are "funeral/begrafenis," "grandma/ oma," "playground/speeltuin," and "resit/hertentamen." The L2 language context words are "airport/luchthaven," "career/ loopbaan," "passion/hartstocht," and "world cup/wereldbeker." 


\section{Results}

For all participants, the emotionality scores of the words in each language and language context were averaged to form four indexes. These variables were then subjected to a repeated-measures ANOVA that also included the language order and word sequence counterbalancing factors and the interaction between them as between-subjects factors. The main effects of language and of language contexts were nonsignificant ( $p$ 's $>.33$ ). The two-way interaction between these factors was significant and in the predicted direction $(F(1,86)=84.03, p<.0001$; see table 2 and fig. 3$)$.

When the language context was predominantly L1, the L1 words $(M=6.12)$ were perceived as more emotional than the L2 words $(M=5.04 ; F(1,86)=50.90, p<.0001)$. Conversely, when the language context was predominantly L2, the L1 words $(M=5.10)$ were perceived as less emotional than the L2 words $(M=6.03 ; F(1,86)=76.35$, $p<.0001)$. At the word level, the effect of language on emotionality was significant and in the predicted direction for all the words in both language context conditions (all $p$ 's <.0001). A number of theoretically uninteresting effects related to the counterbalancing factors and gender (e.g., the language context by gender interaction) were also significant.

\section{Discussion}

In this experiment, we provide direct evidence for the role of the context of language use for the effect of language on emotionality obtained in the previous studies. A set of L1/ L2 word pairs was selected based on a pretest to differentiate between concepts that had been predominantly encountered in L1 versus L2 language contexts. Participants in the main study then rated the emotionality of both L1 and L2 words from each pair. We observed a crossover interaction between language and language context. For concepts encountered predominantly in L1 language contexts, we replicated the emotional advantage of L1 reported in previous experiments, but for concepts encountered predominantly in L2 language contexts, we observed a reversal of this effect: in this case L2 words were rated as more emotional than L1 words.

\section{STUDY 5}

Study 5 was designed to provide additional process evidence for the theory by focusing on the indirect route to the emotionality of L 2 words. In study 3, we showed that the emotionality of L2 words increases when the indirect route is facilitated. Likewise, the emotionality of L2 words should decrease when the indirect route is inhibited. Study 5 tested this prediction with a procedure similar to that of study 4 but relying only on words for which the context of language use is predominantly L1. Participants rated the emotionality of words in L1 and L2. Half were instructed to focus on personal experiences with each target word (i.e., experiences in $\mathrm{L} 1$ contexts for $\mathrm{L} 1$ words and experiences in L2 contexts for L2 words; same-language episodic mem-
TABLE 2

\begin{tabular}{|c|c|c|c|c|}
\hline \multirow{3}{*}{$\begin{array}{l}\text { Emotional } \\
\text { intensity }\end{array}$} & \multicolumn{2}{|c|}{ Study 4} & \multicolumn{2}{|c|}{ Study 5} \\
\hline & \multicolumn{2}{|c|}{$\overline{\text { Language context }}$} & \multicolumn{2}{|c|}{ Instructions } \\
\hline & L1 & L2 & SLEMF & Control \\
\hline L1 & $\begin{array}{c}6.12 \\
(1.11)\end{array}$ & $\begin{array}{c}5.10 \\
(1.17)\end{array}$ & $\begin{array}{l}5.44 \\
(.98)\end{array}$ & $\begin{array}{c}5.46 \\
(1.11)\end{array}$ \\
\hline L2 & $\begin{array}{c}5.04 \\
(1.38)\end{array}$ & $\begin{array}{c}6.03 \\
(1.03)\end{array}$ & $\begin{array}{c}4.31 \\
(1.18)\end{array}$ & $\begin{array}{c}5.10 \\
(1.17)\end{array}$ \\
\hline
\end{tabular}

ory focus condition). The remainder read the same instructions used in study 4 (control condition). This instruction manipulation was designed to disentangle the effect of L1 and L2 memories on the subsequent word-rating task. In particular, relative to the control condition, the same-language episodic memory focus condition should discourage the indirect route, reducing the perceived emotionality of L2 words and, as a consequence, leading to a larger emotional advantage of L1. The emotionality of L1 words should be unaffected by the same-language episodic memory focus because the emotionality of L1 words should always be determined by L1 episodic memories, regardless of instructions.

\section{Method}

Design and Participants. The design of this experiment was a 2 (language: L1 vs. L2) $\times 2$ (instructions: samelanguage episodic memory focus vs. control) mixed design with language manipulated within subjects and instructions between subjects. As in study 4, we also counterbalanced between subjects the order of language presentation and word order. A total of 16 different versions of a booklet were randomly distributed across respondents. Participants were 91 undergraduate students at Erasmus University who were Dutch native speakers and fluent speakers of English (for age, $M=20.02 ; \mathrm{SD}=1.94 ; 55$ males).

Procedure. The study took place in a behavioral lab where participants were asked to fill out a short booklet within a series of unrelated studies. The procedure was similar to that used in study 4. Eight concepts were presented sequentially, and for each of them participants were asked to rate the perceived emotionality of the L1 (Dutch) and L2 (English) words. The first page included the instruction manipulation. In the control condition, the instructions were identical to those administered in study 4 . In the samelanguage episodic memory focus condition, participants were told that "previous research has shown that for people who speak more than one language, whether a word is presented in their native language or in a foreign language can trigger different personal memories" and that, as a consequence, "the English and Dutch words presented in the fol- 
FIGURE 3

LANGUAGE BY LANGUAGE CONTEXT INTERACTION FOR EMOTIONAL INTENSITY IN STUDY 4

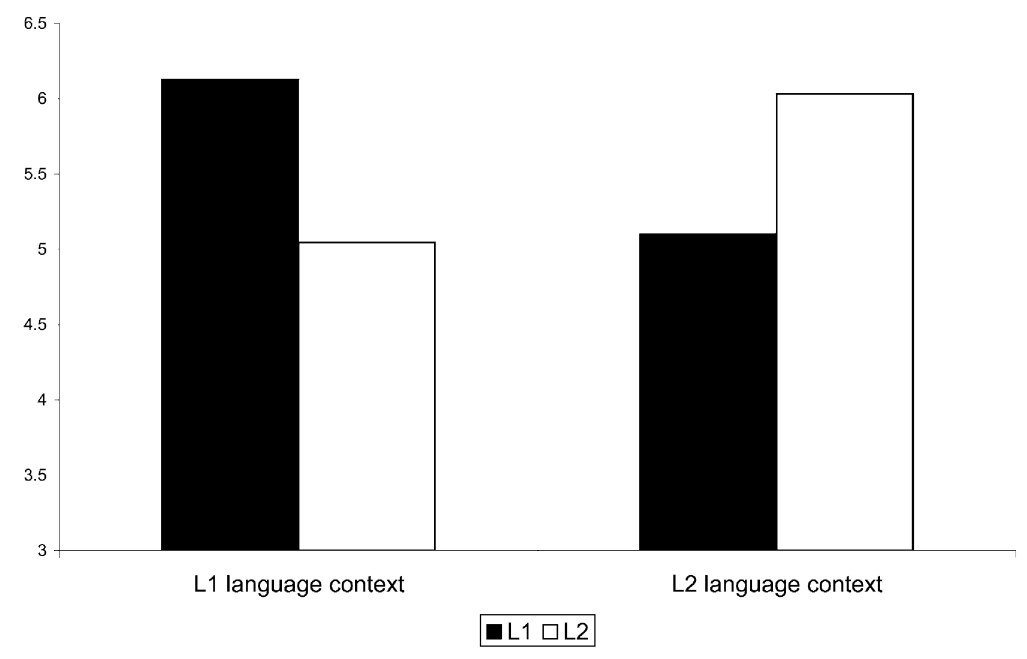

lowing pages can bring to mind different memories." They were then instructed to focus on their personal experiences with the words when rating them. The final page of the survey included some demographic questions and an openended prompt in which participants were asked to guess the purpose of the study.

To select the stimuli, we used the ratings of the prevalence of L1 versus L2 context of language use obtained in the pretest of study 4. To the four pairs of words used in study 4, we added four more by selecting the subsequent pairs in the ranked order of 24 concepts ("farewell/vaarwel," "immature/ onvolwassen," "sheep/schaap," and "Sunday/zondag").

\section{Results}

For each respondent, we calculated two scores by averaging the emotionality of the eight L1 and L2 words. These scores were entered in a repeated-measures ANCOVA with language as a within-subjects factor and instructions as a between-subjects factor. As in study 4, the counterbalancing conditions and their interaction were added as covariates. The results are in line with the research hypotheses (see table 2 and fig. 4).

As predicted, we found a main effect of language $(F(1,82)=47.91, p<.0001)$. The L1 words $(M=5.45)$ were rated as more emotional than L2 words $(M=4.71)$. Follow-up contrast analyses showed this emotional advantage of L1 over L2 to be statistically significant in both the control condition $(F(1,82)=5.69, p<.05$; replicating the results in the L1 language context condition of study 4) and the same-language episodic memory focus condition $(F(1,82)=53.62, p<.0001)$. Importantly, we observed a significant language by instructions interaction $(F(1,82)=$ $13.30, p<.001)$. As predicted, the emotional advantage of L1 over L2 was larger when participants were instructed to focus on same-language episodic memories $(M=1.13)$ than in the control condition $(M=0.36)$. Specifically, the significant interaction was driven by the drop in emotionality of L2 words in the same-language episodic memory focus condition $(M=4.31)$, compared to the control condition $(M=5.10 ; F(1,82)=11, p<.01)$. The instruction manipulation did not affect scores for the L1 words $(p>.85)$.

As a consequence of the nature of the language by instructions interaction (and, in particular, of the drop in the emotionality of L2 words), the main effect of the instructions manipulation was also significant $(F(1,82)=4.44$, $p<.05)$. In the same-language episodic memory focus condition $(M=4.87)$ emotionality ratings were on average lower than in the control condition $(M=5.28)$. The only other significant coefficient in the model was the theoretically uninteresting main effect of order of language presentation. An alternative model was estimated including gender as an additional between-subjects factor. No coefficient involving this variable was, however, significant.

The open-ended responses about the purpose of the study were analyzed to assess the possibility of demand effects. Unsurprisingly, given the procedure, a substantial minority of respondents (31 out of 91) made some sort of reference to a link between language and emotionality. If the moderation of instructions was a consequence of demand effects in the same-language episodic memory focus condition, we should expect references to a link between emotions and language to be more prevalent in this than in the control condition. Contrary to this alternative account, the number of such references was directionally larger in the control condition (18 vs. 13), although this difference was nonsignificant $(p>.30$; the language by instructions interaction remains significant when these participants are excluded from the analysis). In addition, none of the participants in the same-language episodic memory focus referred to the same-language episodic memory focus in any way. Thus, it seems highly unlikely that the main effect of language 
FIGURE 4

LANGUAGE BY INSTRUCTIONS INTERACTION FOR EMOTIONAL INTENSITY IN STUDY 5

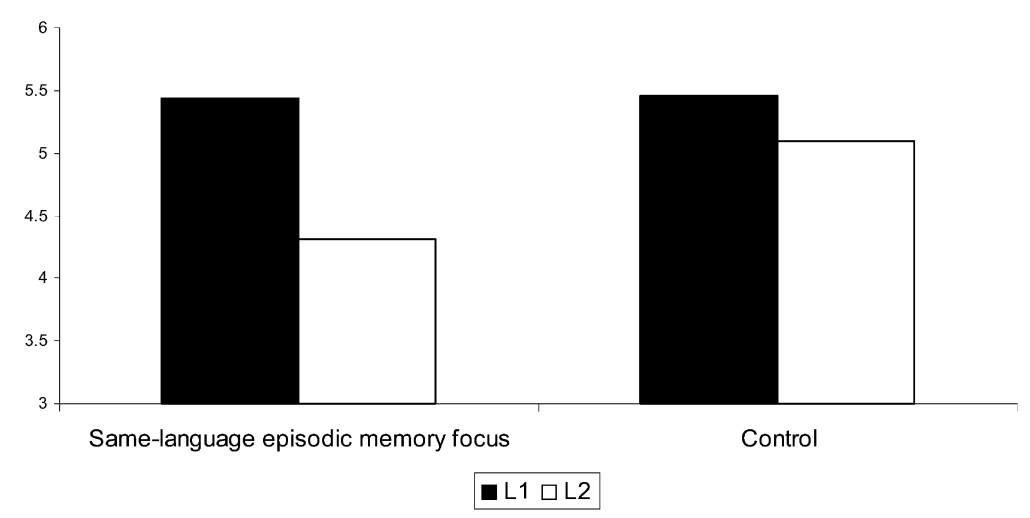

and its interaction with same-language episodic memory focus were due to experimental demand.

\section{Discussion}

This experiment employed a procedure similar to that used in study 4 . In this study we focused only on words with a predominantly L1 context of use and added a manipulation of the instructions to provide additional evidence for the theory. Half of participants were asked to focus on same-language episodic memories when rating the emotionality of a series of L1 and L2 word pairs. We replicated the main effect of language found in studies 1-3. The L1 words were rated as more emotional than L2 words. In this study, moreover, the emotional advantage of L1 was stronger in the presence of instructions asking to focus on personal experiences with the L1 and L2 words.

\section{GENERAL DISCUSSION}

This article presented a language-specific trace theory of language emotionality that extends episodic trace theories (e.g., Hintzman 1986) to issues of language and emotional intensity. Due to the language specificity of episodic memory and the difference between L1 and L2 in frequency of use, the theory predicts that L1 marketing messages generally tend to be perceived by consumers as more emotional than L2 marketing messages.

Across five experiments, we found converging evidence for the theory. Support for the theory was found while ruling out explanations based on country stereotypes (study 1) and comprehension effects (studies 2-3). In addition, the experiments provide process insight through moderation. The effect of language on perceived emotionality was found to be a function of the context of language use (study 4) and of experimental instructions (study 5). The studies also provide strong evidence of external validity. In study 1 , the effect of language was assessed relying on French/Dutch comparisons. It was also assessed using English as L2 and over 20 different L1s (study 3) or Dutch as L1 (studies 2,
4, and 5). Dutch is the language that, from both syntactic and lexical points of view, is closest to English (Finegan 1987). As degree of language overlap determines the activation of the L1 representation in processing L2 words (Sunderman and Kroll 2006), this points to an especially conservative test of the theory. Finally, support for the theory was found using a variety of stimuli.

The language-specific episodic trace theory of language emotionality contributes to the current understanding of the emotional intensity of language for bilinguals by integrating and extending psycholinguistic perspectives in this area. The theory draws from the Revised Hierarchical Model (Kroll and Stewart 1994) in its consideration of separate lexical stores for L1 and L2 and in the asymmetric relationship between them. The theory borrows from literature on language-dependent recall and on the emotions of bilinguals (Altarriba 2003; Harris et al. 2006; Marian and Kaushanskaya 2004; Marian and Neisser 2000; Pavlenko 2005) in the emphasis on the role of episodic and autobiographical memories in determining the emotionality of L1 and L2 words.

The theory also adds to the cognitive literature in two main ways. First, it adds to literature on episodic trace models by pointing out the pivotal role played by emotional echo content in episodic traces. Second, while this literature has exclusively focused on memory accessibility, we extend the application of episodic trace models to the issue of emotionality. As such, this theory could be useful to explore issues of emotionality beyond the bilingual setting. For example, the relationship between the frequency of using a word and emotionality should hold also in a monolingual setting.

Furthermore, this article makes a number of contributions specific to consumer research. From a substantive point of view, the implication of our findings is that, ceteris paribus, it is generally preferable to communicate with consumers using their own native language, as doing so should result in more emotional messages. From a broader point of view, this article is the first to adopt a psycholinguistic perspective 
on the emotional consequences of the process of globalization for consumers. Bilingualism is a growing area in consumer research (Luna and Peracchio 2001, 2005; Luna, Ringberg, and Peracchio 2008; Tavassoli and Lee 2003), and the article adds a new dimension to this body of literature. We identify two main directions along which the current theorizing and findings could be extended in future research.

\section{Second-Order Consequences of Language Emotionality}

The language effects on emotionality explored in this article are likely to have important second-order consequences for consumer behavior. The decision-making literature shows that affective and cognitive factors have separate influences on consumer decisions (Epstein 1994; Loewenstein et al. 2001). Our findings suggest that, when emotional factors are important in decision making, the language in which options are framed may exert an important influence on product choice. For example, when products differ in terms of their emotional versus more cognitive benefits (e.g., taste experience vs. health consequences in chocolate cake vs. fruit salad; Shiv and Fedorikhin 1999), the extent to which the emotional benefits really "hit home" at an emotional level may depend on whether those benefits are described in L1 versus L2. As a result, the impact of emotional benefits relative to more cognitive benefits might be higher in L1 than L2. This might imply that self-control, in the sense of passing up an immediate emotional benefit for a longer-term cognitive benefit, might be more difficult in L1 than L2 contexts.

Furthermore, Rottenstreich and Hsee (2001) showed that small probabilities of obtaining emotional outcomes are overweighted more than small probabilities for less emotional outcomes such as money. This research suggests that the curvature in Prospect Theory's (Kahneman and Tversky 1979) weighting function becomes more extreme as choice outcomes are processed in a more emotional way. Our results imply that choice outcomes will be experienced in a more emotional manner if they are described in L1 than in L2. Thus, it is possible that consumers' weighting functions show more curvature in consumers' native language than in their second language. To the extent that nonlinearity in Prospect Theory's value function is also a function of emotionality (cf. Hsee and Rottenstreich 2004), a similar effect might occur with respect to the value function. That is, consumers may be more risk averse for gains, more loss averse, and more risk seeking for losses and show stronger endowment effects in L1 than in L2.

In sum, consumers' decision processes might be different in L1 versus L2 contexts. "Hot" emotional processes might play a larger role, and emotional benefits might weigh more heavily relative to "cold" processes and more cognitive benefits in consumers' native than in their second language, yielding different preferences and choices.

\section{Generalizations Outside the Emotional Domain}

Whereas this article considered the relevance of the language-specific trace theory for emotional appraisals, future research should explore the generality of the theory in the cognitive domain. For example, in line with the finding that a bilingual's native language may activate more thoughts about one's family and friends (Noriega and Blair 2008), different languages may also trigger different echoes of brand names and product categories that are routinely stored as a part of consumption episodes. For example, for a bilingual consumer who has drunk, or seen ads, for beers in English- versus Dutch-language contexts, the word "beer" might activate experiences involving different brands of beer (e.g., Budweiser) than the word "bier" (e.g., Hertog Jan). Thus, different brands may be part of a consumer's consideration set in different-language contexts. Future research should investigate under which circumstances this differential memory trace activation influences preferences and behavior (e.g., stimulus-based choice vs. memory-based choice) and to what extent this influence is conscious or unconscious in nature.

Consumers may also weight product attributes differently in $\mathrm{L} 1$ and $\mathrm{L} 2$ contexts. For example, if in a product category, advertisements, personal conversations, and media coverage in one language tend to mention an attribute more often in one language than another, consideration of the product category would yield echoes that highlight different attributes in different languages that should affect the weight of these attributes in the different languages. For example, if the word "yogurt" activates episodes focusing on nutritional benefits in one language but on taste in another language, the same consumer is likely to place a higher weight on healthiness and a lower weight on taste in one language than in the other. Thus, the same consumer in an otherwise similar situation may buy yogurt containing extra probiotics, or "friendly" bacteria, in one language context and a more fullflavored yogurt with extra strawberries in the other language context.

In sum, the language-specific trace theory has broad implications beyond emotionality. Language-specific traces of experiences should contain not only emotions but also brand names, product attributes, product categories, and behaviors. This allows words in different languages to probe different brand names, product attributes, product categories, and behaviors, leading consumers to consider and choose different products. Whereas these predictions follow directly from the same theory that led to the general recommendation to advertise in consumers' native language, they also suggest additional boundary conditions of the generally positive effect of advertising in L1. If advertising in L2 generates echoes that include one's own brand or that highlight attributes that are a strength of one's own brand, advertising in L1 may be a mistake.

\section{Conclusion}

Globalization is a defining social trend of our times and "one of the dominant forces in the psychological develop- 
ment of the people in the 21st century" (Arnett 2002, 781). The importance of this process demands that consumer researchers begin to address issues directly related to globalization, such as bilingualism (Johar et al. 2006). This article represents a step in this direction by providing insight into the consequences for emotional processes of the globalization of advertising language and of the increasing use of English in ads from countries that do not have English as their first language.

\section{REFERENCES}

Altarriba, Jeanette (2003), "Does Carino Equal 'Liking'? A Theoretical Approach to Conceptual Nonequivalence between Languages," International Journal of Bilingualism, 7 (3), 305-22.

Arnett, Jeffrey J. (2002), "The Psychology of Globalization," American Psychologist, 57 (10), 774-83.

Bloise, Suzanne M. and Marcia K. Johnson (2007), "Memory for Emotional and Neutral Information: Gender and Individual Differences in Emotional Sensitivity," Memory, 15 (2), 192-204.

Bond, Michael H. and Tat-Ming Lai (1986), "Embarrassment and Code-Switching into a Second Language," Journal of Social Psychology, 126 (2), 179-86.

Bower, Gordon H. (1981), "Mood and Memory," American Psychologist, 36 (2), 129-48.

Costa, Albert, Alfonso Caramazza, and Nuria Sebastian-Galles (2000), "The Cognate Facilitation Effect: Implications for Models of Lexical Access," Journal of Experimental Psychology: Learning, Memory, and Cognition, 26 (5), 1283-96.

Cristal, David (1997), English as a Global Language, Cambridge: Cambridge University Press.

Davis, Penelope J. (1999), "Gender Differences in Autobiographical Memory for Childhood Emotional Experiences," Journal of Personality and Social Psychology, 76 (3), 498-510.

Dewaele, Jean-Marc (2004), "The Emotional Force of Swearwords and Taboo Words in the Speech of Multilinguals," Journal of Multilingual and Multicultural Development, 25 (2-3), 204-22.

Epstein, Seymour (1994), "Integration of the Cognitive and the Psychodynamic Unconscious," American Psychologist, 49 (8), 709-24.

Finegan, Edward (1987), "English," in The World's Major Languages, ed. Bernard Comrie, Beckenham: Croom Helm, 77-109.

Gerritsen, Marinel, Hubert Korzilius, Frank van Meurs, and Inge Gijsbers (2000), "English in Dutch Commercials: Not Understood and Not Appreciated," Journal of Advertising Research, 40 (4), 17-31.

Harris, Catherine L., Ayse Aycicegi, and Jean Berko Gleason (2003), "Taboo Words and Reprimands Elicit Greater Autonomic Reactivity in a First Language than in a Second Language," Applied Psycholinguistics, 24 (4), 561-79.

Harris, Catherine L., Jean Berko Gleason, and Ayse Aycicegi (2006), "When Is a First Language More Emotional? Psychophysiological Evidence from Bilingual Speakers," in $B i$ lingual Minds: Emotional Experience, Expression, and Representation, ed. Aneta Pavlenko, Clevedon: Multilingual Matters, 257-311.

Harris, Paul L. (2000), "Understanding Emotion," in Handbook of Emotions, ed. Michael Lewis and Jeanette M. Haviland-Jones, New York: Guilford, 281-92.
Hintzman, Douglas L. (1986), “'Schema Abstraction' in a Multiple-Trace Memory Model," Psychological Review, 93 (4), 411-28.

(1988), "Judgments of Frequency and Recognition Memory in a Multiple-Trace Memory Model," Psychological Review, 95 (4), 528-51.

Hsee, Christopher K. and Yuval Rottenstreich (2004), "Music, Pandas, and Muggers: On the Affective Psychology of Value," Journal of Experimental Psychology: General, 133 (1), 23-30.

Jacoby, Larry L. and Lee R. Brooks (1984), "Nonanalytic Cognition: Memory, Perception, and Concept Learning," in The Psychology of Learning and Motivation, ed. Gordon $\mathrm{H}$. Bower, New York: Academic Press, 1-47.

Jacoby, Larry L. and C. A. Gordon Hayman (1987), "Specific Visual Transfer in Word Identification," Journal of Experimental Psychology: Learning, Memory, and Cognition, 13 (3), 456-63.

Javier, Rafael A. (1989), "Linguistic Considerations in the Treatment of Bilinguals," Psychoanalytic Psychology, 6 (1), 87-96.

Javier, Rafael A., Felix Barroso, and Michele A. Munoz (1993), "Autobiographical Memory in Bilinguals," Journal of Psycholinguistic Research, 22 (3), 319-38.

Johar, Gita V., Durairaj Maheswaran, and Laura A. Peracchio (2006), "MAPping the Frontiers: Theoretical Advances in Consumer Research on Memory, Affect, and Persuasion," Journal of Consumer Research, 33 (1), 139-49.

Kahneman, Daniel and Amos Tversky (1979), "Prospect Theory: An Analysis of Decision under Risk," Econometrica, 47 (2), 263-91.

Koslow, Scott, Prem N. Shamdasani, and Ellen E. Touchstone (1994), "Exploring Language Effects in Ethnic Advertising: A Sociolinguistic Perspective," Journal of Consumer Research, 20 (4), 575-85.

Kroll, Judith F. and Erika Stewart (1994), "Category Interference in Translation and Picture Naming: Evidence for Asymmetric Connections between Bilingual Memory Representations," Journal of Memory and Language, 33 (2), 149-74.

Leclerc, France, Bernd H. Schmitt, and Laurette Dubé (1994), "Foreign Branding and Its Effects on Product Perceptions and Attitudes," Journal of Marketing Research, 31 (2), 263-70.

Lee, Jamie S. (2006), "Linguistic Constructions of Modernity: English Mixing in Korean Television Commercials," Language in Society, 35 (1), 59-91.

Loewenstein, George F., Elke U. Weber, Christopher K. Hsee, and Ned Welch (2001), "Risk as Feelings," Psychological Bulletin, 127 (2), 267-86.

Luna, David and Laura A. Peracchio (2001), "Moderators of Language Effects in Advertising to Bilinguals: A Psycholinguistic Approach," Journal of Consumer Research, 28 (2), 284-95.

(2005), "Advertising to Bilingual Consumers: The Impact of Code-Switching on Persuasion," Journal of Consumer Research, 31 (4), 760-65.

Luna, David, Torsten Ringberg, and Laura A. Peracchio (2008), "One Individual, Two Identities: Frame Switching among Biculturals," Journal of Consumer Research, 35 (2), 279-93.

Marian, Viorica and Margarita Kaushanskaya (2004), "Self-Construal and Emotion in Bicultural Bilinguals," Journal of Memory and Language, 51 (2), 190-201.

Marian, Viorica and Ulric Neisser (2000), "Language-Dependent Recall of Autobiographical Memories," Journal of Experimental Psychology: General, 129 (3), 361-68.

Noriega, Jaime and Edward Blair (2008), “Advertising to Bilin- 
guals: Does the Language of Advertising Influence the Nature of Thoughts?" Journal of Marketing, 72 (5), 69-83.

Palmeri, Thomas J., Stephen D. Goldinger, and David B. Pisoni (1993), "Episodic Encoding of Voice Attributes and Recognition Memory for Spoken Words," Journal of Experimental Psychology: Learning, Memory, and Cognition, 19 (2), 309-28.

Pavlenko, Aneta (2005), Emotions and Multilingualism, Cambridge: Cambridge University Press.

- ed. (2006), Bilingual Minds: Emotional Experience, Expression, and Representation, Clevedon: Multilingual Matters.

Raaijmakers, Jeroen G. W. and Richard M. Shiffrin (1992), "Models for Recall and Recognition," Annual Review of Psychology, 43, 205-34.

Rottenstreich, Yuval and Christopher K. Hsee (2001), "Money, Kisses, and Electric Shocks: On the Affective Psychology of Risk," Psychological Science, 12, 185-90.

Schacter, Daniel L. and Barbara A. Church (1992), "Auditory Priming: Implicit and Explicit Memory for Words and Voices," Journal of Experimental Psychology: Learning, Memory, and Cognition, 18 (5), 915-30.

Schimmack, Ulrich and Rainer Reisenzein (1997), "Cognitive Pro- cesses Involved in Similarity Judgments of Emotions," Journal of Personality and Social Psychology, 73 (4), 645-61.

Shiv, Baba and Alexander Fedorikhin (1999), "Heart and Mind in Conflict: The Interplay of Affect and Cognition in Consumer Decision Making," Journal of Consumer Research, 26 (3), 278-92.

Siemer, Matthias and Rainer Reisenzein (2007), "The Process of Emotion Inference," Emotion, 7 (1), 1-20.

Sunderman, Gretchen and Judith F. Kroll (2006), "First Language Activation during Second Language Lexical Processing: An Investigation of Lexical Form, Meaning, and Grammatical Class," Studies in Second Language Acquisition, 28 (3), 387-422.

Tavassoli, Nader T. and Yih Hwai Lee (2003), “The Differential Interaction of Auditory and Visual Advertising Elements with Chinese and English," Journal of Marketing Research, 40 (4), 468-80.

Tulving, Endel and Donald M. Thomson (1973), "Encoding Specificity and Retrieval Processes in Episodic Memory," Psychological Review, 80 (5), 352-73. 